\title{
A REVIEW ON REVERSIBLE LOGIC GATES
}

\author{
Galadima ${ }^{1}$,B.Y., Galadanci, G.S.M., ${ }^{1}$ Tijjani, A. ${ }^{1}$ and Ibrahim, M. ${ }^{2}$ \\ ${ }^{1}$ Department of Physics, Bayero University, Kano, Nigeria) \\ ${ }^{4}$ Department of Physics, Yusuf Maitama sule university, kano. \\ Corresponding Author: bygaladima.phy@buk.edu.ng
}

ABSTRACT

In recent years, reversible logic circuits have applications in the emerging field of digital signal processing, optical information processing, quantum computing and nano technology. Reversibility plays an important role when computations with minimal energy dissipation are considered. The main purpose of designing reversible logic is to decrease the number of reversible gates, garbage outputs, constant inputs, quantum cost, area, power, delay and hardware complexity of the reversible circuits. This paper reveals a comparative review on various reversible logic gates. This paper provides some reversible logic gates, which can be used in designing more complex systems having reversible circuits and can execute more complicated operations using quantum computers. Future digital technology will use reversible logic gates in order to reduce the power consumption and propagation delay as it effectively provides negligible loss of information in the circuit.

Keywords: Garbage output, Power dissipation, quantum cost, Reversible Gate, Reversible logic,

\section{INTRODUCTION}

In present day technology, Reversible logic has spread its popularity in numerous technologies, due to their ability to reduce the power dissipation which is the main requirement in low power VLSI design (Yelekar \& Chiwande, 2011). Applications of the Reversible circuits can be found in the emerging fields of low power CMOS design, optical information processing, DNA computing, quantum computing and nano technology (Markle \& Drexler, 1996).

Traditional logic circuits are Irreversible which result in energy dissipation and information loss (Biswas et al., 2014). Landaurer's Research has proved that the amount of energy dissipated for every irreversible bit operation is atleast kTLn2 joules, where is $\mathrm{k}$ is the Boltzmann's constant and $T$ is the temperature in Kelvin (Landaurer, 1961; Parhami, 2006). In 1973, Bennett showed that in order to avoid kTLn2 joules of energy dissipation in a circuit it must be built from reversible circuits (Bennett, 1973). The amount of energy dissipated in a system bears a direct relationship to the number of bits erased during computation (Mamataj et al., 2014). Reversible circuits are those circuits that do not lose information.

Reversible logic gates are required to design reversible circuits (Biswas etal., 2014). A reversible logic gate has equal input and output in order to have one to one mapping (Biswas etal., 2014). In reversible circuit there should be Misra, \& Sen, (2017) invented two kinds of novel error control circuits such as hamming no fan-out; that is, each output will be used only once and for each input pattern there should be unique output pattern.

In the year 2007 (James et al., 2007) implement a low power circuit using reversible logic that provides single error correction - double error detection (SEC-DED). The design was done using a new $4 \times 4$ reversible gate called Hamming Code Generating (HCG) for implementing hamming error coding and detection circuits. A parity preserving Hamming Code Generating (PPHCG) that preserves the input parity at the output bits is used for achieving fault tolerance for the hamming error coding and detection circuits.

Rashmi, \& Tilak, (2011) invented a reversible gate known as Binary Coded Decimal subtraction correction logic (BSCL). The main purpose of introducing this gate is either to find correction logic for BCD subtraction or to pass same data to the output.

Misra, \& Wairya, (2015) demonstrated a reversible $B C D$ adder and carry skip $B C D$ adder circuit based on three new type of reversible gates, namely; Full adder subtraction (FAS), Half adder subtraction (HAS) and Overflow detection (OD) gates, to optimize the adder circuits. The new type of reversible full adder using FAS gate is the best circuit in terms of quantum cost. By utilizing those three new types of gates, reversible n-digit $B C D$ adder and 1-digit carry skip BCD adder are proposed with its algorithm. code, parity generator and checker. To design the HG-PP ( $\mathrm{HG}=$ Hamming gate, $\mathrm{PP}=$ Parity 
Special Conference Edition, November, 2019 preserving), NG-PP (NG = New gate) are proposed for optimising the circuits. Based on the proposed gates and few existing gates, the hamming code and parity generator and checker circuits are constructed. The reversible, major metrics such as gate count, quantum cost, unit delay, and garbage outputs, uses best optimisation results compared to counterparts. They can be utilised as a low power error control circuit applied in future communication systems. Bhoi, K.B, \& Misra, N.K. (2017) introduced a new gate named as universal reversible QCA gate (URQG) is proposed. It is a $3 \times 3$ gate that realizes 13 standard functions with optimal gate count. 2. The URQG gate is compared with the existing reversible gates using standard Boolean equations. The proposed gate outperforms the existing gates in terms of design cost and testing overhead. An n-bit comparator, was synthesised where proposed URQG and existing Feynman gates are cascaded together.

\section{BASIC DEFINITIONS OF REVERSIBLE LOGIC}

\section{REVERSIBLE FUNCTION}

A function $f \in B_{n . n}$ is called reversible if $\mathrm{f}$ is bijective, i.e., if each input pattern is uniquely mapped to a corresponding output pattern and vice versa. Otherwise, it is called irreversible. Clearly, if $f$ is reversible, then its number of inputs is equal to the number of outputs. In other words, each reversible function $f \in B_{n . n}$ is a bijection that performs a permutation of the set of input patterns (Abdessied \& Drechler, 2016). An irreversible function can be embedded into a reversible specification by adding extra variables to achieve a bijective function. An embedding is not unique and the choice of embedding can have a very significant effect on the number of the variables of the resulting function (Miller et al., 2009; Soeken et al., 2015).

\section{REVERSIBLE LOGIC GATE}

A reversible logic gate is an n-input n-output logic device with one-to-one mapping (Yelekar \& Chiwande, 2011), the number of inputs are equal to the number of the outputs of the gates in order to have a one-to-one mapping. This generates a unique set of output vector for each set of input vector (Biswas et al., 2014). This prevents the loss of information which causes power dissipation. In reversible logic, fan-out is not possible and feedback or loops are not allowed. Some features of a reversible logic circuit are: Minimum input constants, Minimum number of reversible gates and Minimum number of garbage outputs.

\section{REVERSIBLE CIRCUIT}

A combinational reversible circuit is an acyclic combinational logic circuit in which all gates are reversible, and are interconnected without explicit fan-outs and loops. Boolean functions can be synthesized to a reversible circuit after embedding them to reversible functions. Therefore, in general a reversible circuit contains $\mathrm{n}$ inputs with $\mathrm{p}$ primary inputs and $\mathrm{c}$ constant inputs with $p+c=n$. At the output side, there are $m$ primary outputs and $k$ garbage outputs with $\mathrm{k}+\mathrm{m}=\mathrm{n}$. Figure1 depicts the general structure of a reversible circuit (Abdessied \& Drechler, 2016). A reversible circuit should be designed using minimum number of reversible logic gates, minimum input constant, minimum number of garbage outputs.

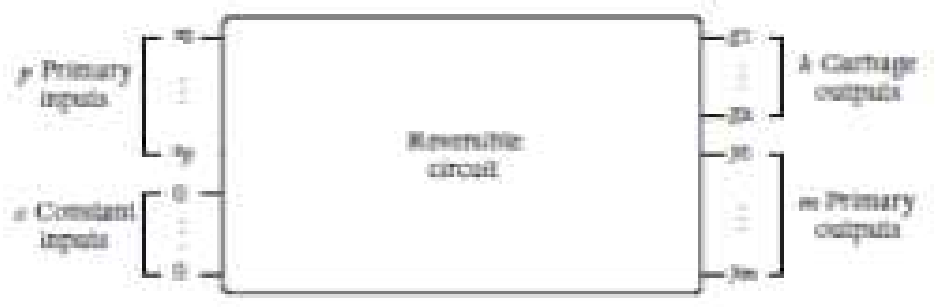

Figure 1: Reversible Circuit Structure

\section{GARBAGE OUTPUT}

Unwanted output of reversible gate is called garbage output. The output of reversible gate is not used as a primary output or as input to other gates is called garbage output. Garbage's outputs are needed in circuit to maintain reversibility concept. Figure 2 shows an example of reversible function $\mathrm{f}=x_{1} x_{2} X O R x_{3}$, the two unused pins are the garbage outputs (Ankush \& Bhandari, 2016). 


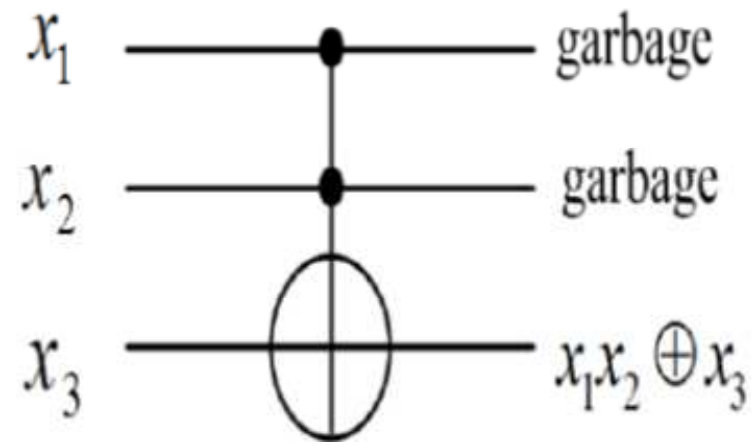

Figure 2: Garbage output

\section{CONSTANT INPUTS}

This can be defined as the number of inputs that are to be maintain constant at either 0 or 1 in order to synthesize the given logical function (Thapiyal \& Ranganathan, 2010).

\section{QUANTUM COST}

Quantum cost may be defined as the cost of the circuit in terms of the cost of a primitive gate. It is calculated by the number of primitive reversible logic gates $(1 \times 1$ or $2 \times 2)$ required to realize the circuit. The quantum cost of a circuit is the minimum number of $2 \times 2$ unitary gates to represent the circuit keeping the output unchanged. The quantum cost of a $1 \times 1$ gate is 0 and that of any $2 \times 2$ gate is the same, which is 1(Smoline \& David, 1996).

\section{DELAY}

The delay of a logic circuit is the maximum number of gates in a path from any input line to any output line. The definition is based on two assumptions: (i) Each gate performs computation in one unit time and (ii) all inputs to the circuit are available before the computation begins. (Mohammadi \& Eshghi, 2009).

\section{HARDWARE COMPLEXITY}

Hardware Complexity refers to the total number of logic operation in a circuit. Means the total number of AND, OR and EXOR operation in a circuit (Akbar et al., 2011).

\section{REVERSIBLE LOGIC GATES}

There are many number of reversible logic gates that exist in present literature. Some of the reversible gates are presented by (Ankush \& Bhandari, 2016). In this review we try to show other reversible gates which are not presented by (Ankush \& Bhandari, 2016) and may be useful to researchers. The reversible gates are given below;

\section{SG Gate}

SG gate is also known as Sayem gate (Sayem\& Ueda, 2010) is a $4 \times 4$ reversible gate. The input and output vector of this gate are, Iv $=(A, B, C$, D) and $\mathrm{OV}$ $=$ $\left(A, A^{\prime} B \oplus A C, A^{\prime} B \oplus A C \oplus D, A B \oplus A^{\prime} C \oplus D\right)$. The block diagram of this gate is shown in Figure 3.

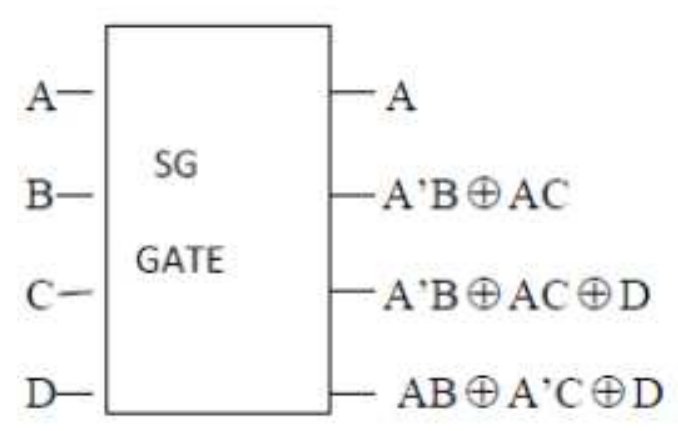

Figure 3: Sayem gate

\section{BME Gate}

BME gate is a $4 \times 4$ reversible gate (Mahfuzzreza et al., 2013). The BME gate can be described as: $I V=(A, B, C, D) ; O V=(A, A B \oplus C$,
$\left.A D \oplus C, A^{\prime} B \oplus C \oplus D\right)$, where IV and OV are input and output vectors respectively. Quantum cost of BME gate is five (Garipelly et al., 2013). Figure 4 shows a $4 \times 4$ BME gate. 


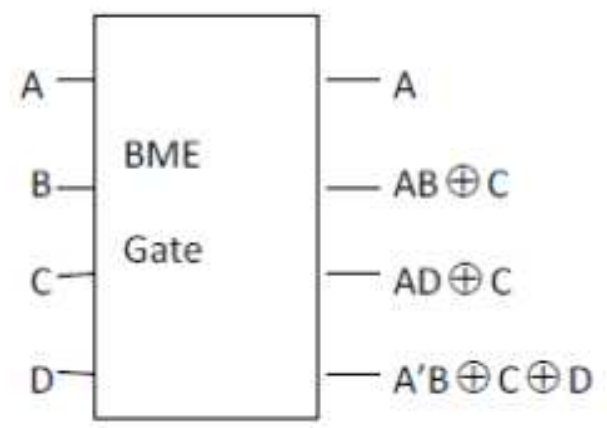

Figure 4: BME Gate

\section{BSCL Gate}

Binary Coded Decimal Subtraction Correction logic gate is a $6 \times 6$ reversible gate. The purpose of this gate is either to find correction logic for BCD subtraction or to pass same data to the output depending on the control signal (Rashmi et $a l ., 2011)$. Here $F$ is the control signal as shown in the figure if $F$ is equal to $0, A, B, C, D$ and $E$ as it is passed to the output $P$ Q R S and $T$. If $F$ is equal to 1 , then output $Q R S$ and $T$ depends on the value of $E$. If $E$ is equal to 0 then $Q R$ S and $T$ is the nines compliment of the input binary number $A B C$ and $D$. If $E$ is equal to 1 then binary number 0001 is added to $A B C D$ to get the valid corrected subtraction result.

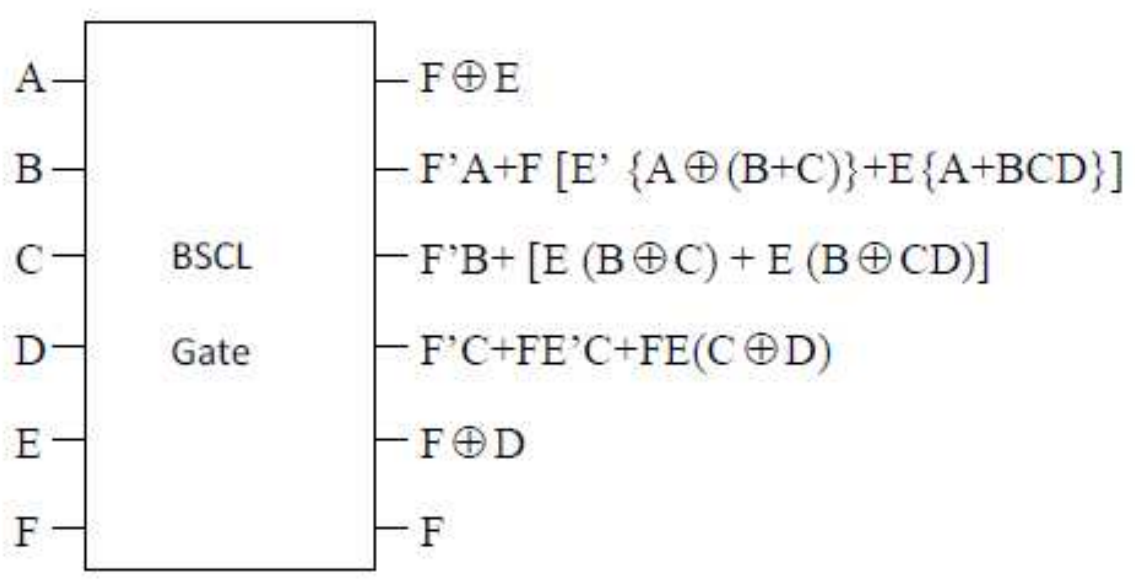

PTR Gate

Figure 5: BSCL Gate

PTR gate is a $4 \mathrm{X} 4$ Reversible gate that can work as a reversible full adder

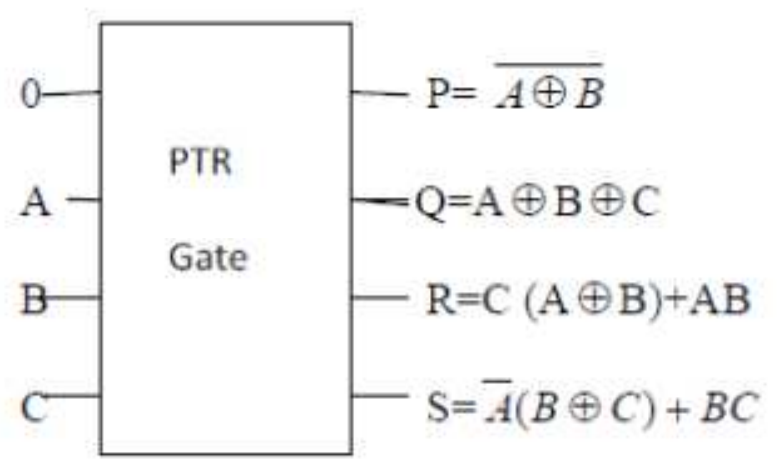

Figure 6: PTR Gate

\section{PAREEK GATE}

PAREEK gate is a $4 \times 4$ reversible gate (Pareek et al., 2014). The PAREEK gate can be described as: $\mathrm{IV}=(\mathrm{A}, \mathrm{B}, \mathrm{C}, \mathrm{D}) ; \mathrm{OV}=\left(\mathrm{A}, \mathrm{A}^{\prime} \mathrm{B} \oplus \mathrm{AD}\right.$, $\left.A^{\prime} B \oplus A D \oplus C, B \oplus D\right)$, where IV and OV are input and output vectors respectively. Quantum cost of PAREEK gate is seven which is illustrated in (Pareek et al., 2014). Figure 7 shows a $4 \times 4$ PAREEK gate. 
RCQCA GATE

Reversible Conservative quantum dot Cellular Automata gate is a $4 \times 4$ reversible gate (Misra

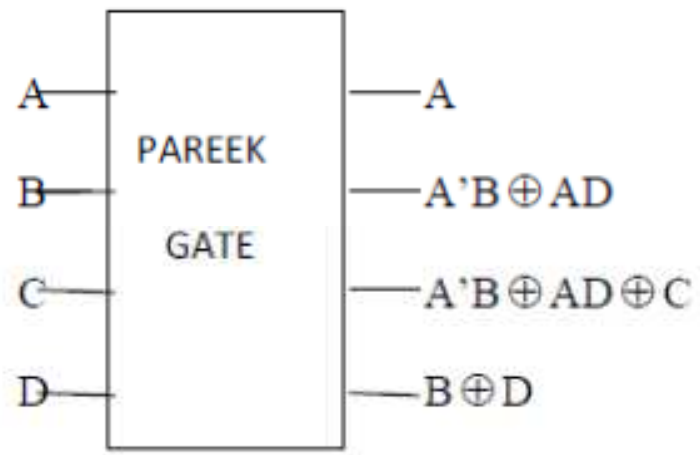

Figure 7: Pareek Gate

et al., 2018). Quantum cost of 6. This gate is utilised for Sequential circuit synthesis of reversible

functions

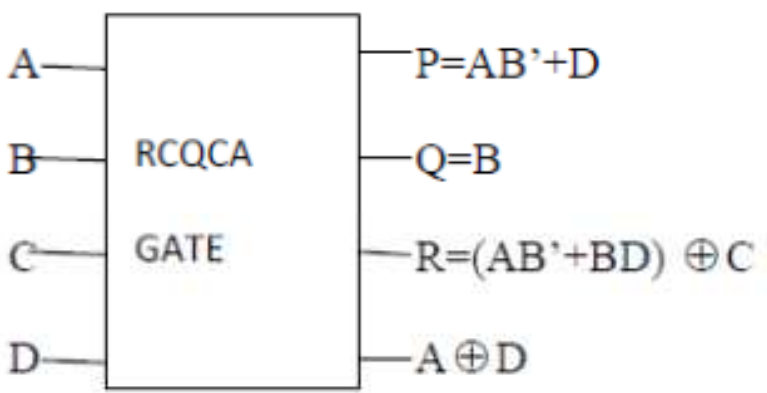

Figure 8: RCQCA Gate

\section{URQG GATE}

Universal Reversible Quantum dot Cellular Automata Gate is a $3 \times 3$ reversible gate (Bhoi et al., 2017). Based on reversibility given the outputs logic $P, Q, R$ the inputs $A, B, C$ can be computed. The reversible URQG gate is a universal gate and can implements 13 standard functions. By combining two URQG gate N-bit comparator can designed.

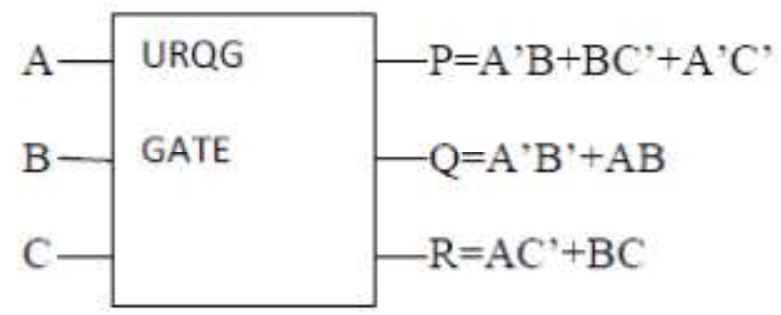

Figure 9: URQG Gate

\section{HCG GATE}

A $4 \times 4$ reversible gate, Hamming Code Generating gate (James et al., 2007) is depicted in Figure 10. HCG gate is one-through gate which means that one of the input variables is also output. Hamming error coding and detection circuit can be implemented using this gate

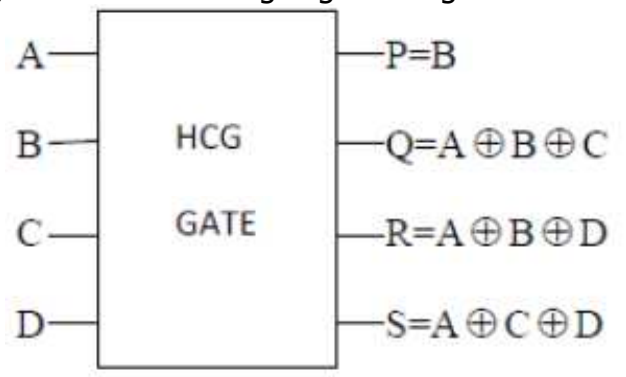

Figure 10: HCG Gate 
Special Conference Edition, November, 2019 HAS GATE

Half Adder Subtraction gate is a $3 \times 3$ reversible gate which is helpful for the design of reversible BCD adder and carry skip BCD adder circuit. The HAS gate has a quantum cost 5 . It consist of four XOR gates, two controlled- $V$ and one controlled $-\mathrm{V}^{+}$gate. This gate can implement the operation of haif adder and full subtraction. (Misra et al., 2015).

\section{FAS GATE}

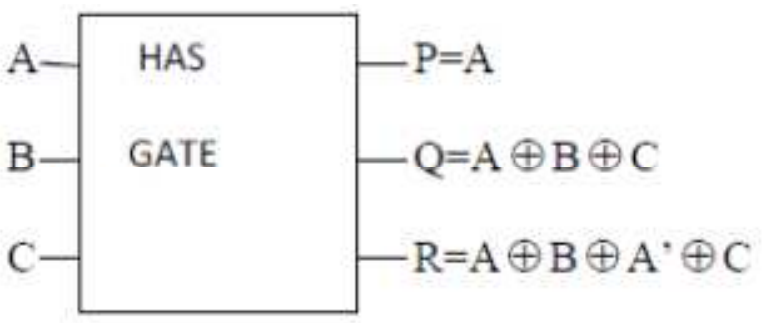

Figure 11: HAS Gate
Full Adder Subtraction gate is a $4 \times 4$ reversible gate. FAS gate can perform the operation of full adder and full subtraction. The gate has a quantum cost of 8 . It consist of six XOR gates, two controlled- $\mathrm{V}$ and one controlled- $\mathrm{V}^{+}$. (Misra et

al.,

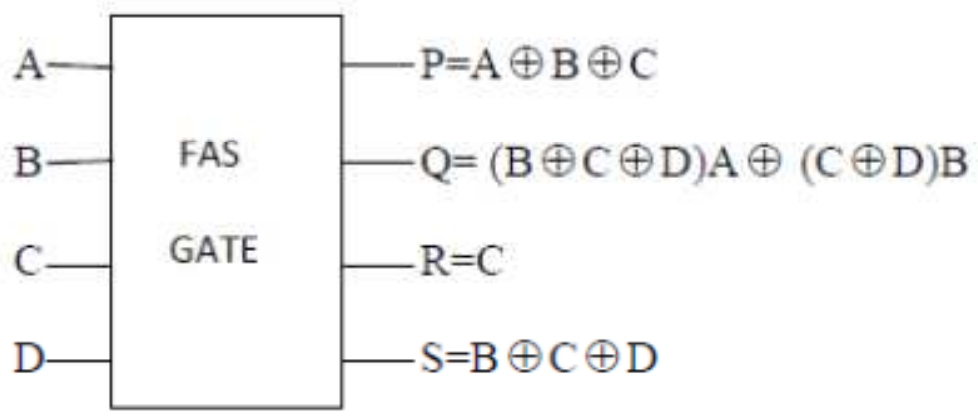

Figure 12: FAS Gate

OD GATE

Over flow Detection gate is a $5 \times 5$ reversible gate. The OD gate has a quantum cost of 10 . It consist of seven XOR gates, four controlled-V and two controlled- $\mathrm{V}^{+}$gate. OD gate is used for overflow detection. (Misra et al., 2015).

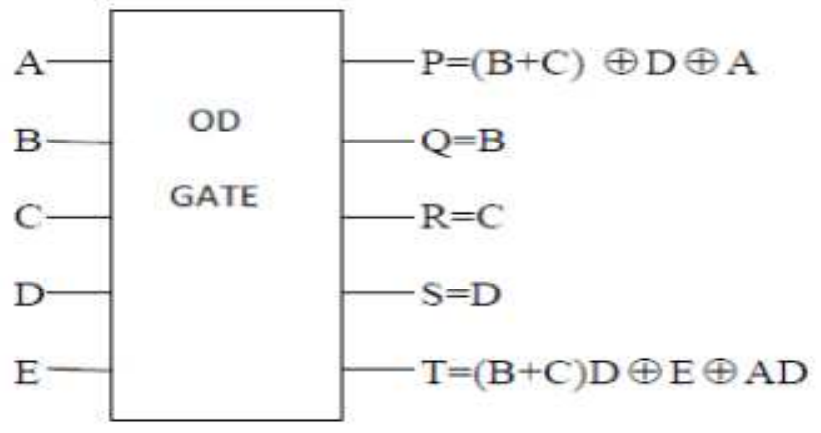

Figure 13: OD Gate

\section{PPHCG GATE}

Parity Preserving Hamming Code Generating gate is a $4 \times 4$ Parity Preserving Reversible gate. The outputs preserve the input parity (James et al., 2007).This gate can be used for achieving fault tolerance for the hamming error coding and detection. 


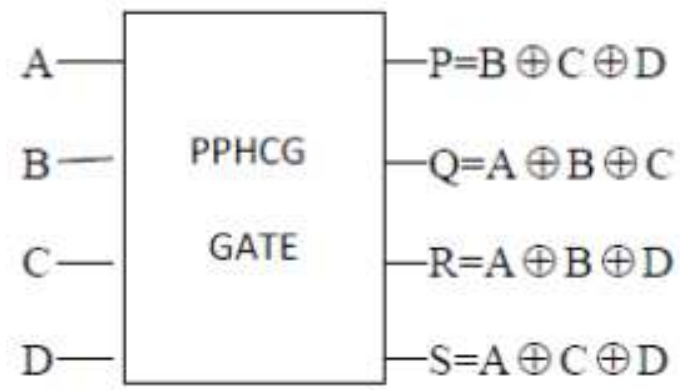

Figure 14: PPHCG Gate.

NG-PP

The NG-PP structure is utilised of 5-input and 5output. The input parity to output parity is conserved. Hence this gate is a conservative gate. Further, it holds the bijective mapping, it also the reversible gate. The QC of NG-PP gate is 5(Misra et al., 2017). It can singly perform the logic operation of parity generator and checker.

\section{HG-PP GATE}

A $5 \times 5$ conservative reversible logic gate named HG-PP is shown in Figure16.. It depicts the same count of 1 's in the output as well as input, further maintain the bijective-mapping property

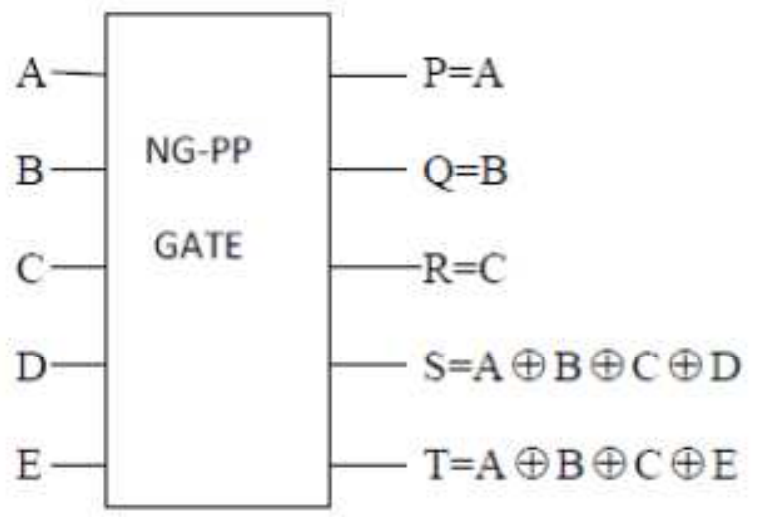

Figure 15: NG-PP Gate

of the reversibility. Hence this gate is reversible as well as conservative. The QC of HG-PP gate is 4(Misra et al., 2017). This gate is helpful for the design of hamming code.

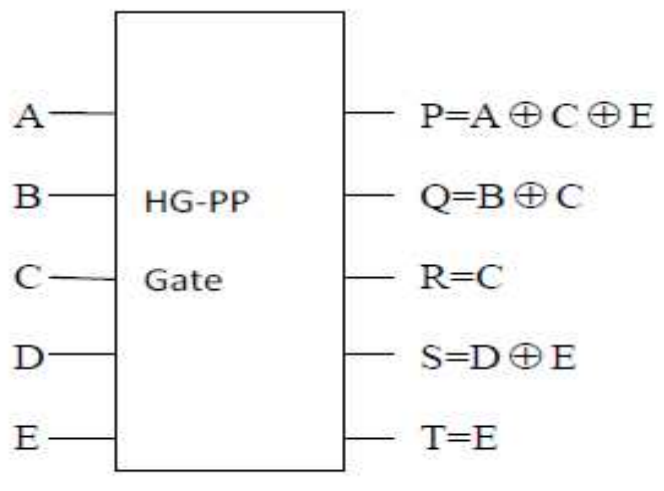

Figure 16: HG-PP Gate

\section{COMPARATIVE STUDY}

Various reversible gates and different circuits associated with these gates are discussed here. And also comparisons have been made among the existing circuit in terms of various parameters such as quantum cost, garbage output, constant input, gate count and delay. Comparison between existing reversible gates is shown in Table 1. 
Special Conference Edition, November, 2019

Table 1: Comparison between Reversible Logic Gates

\begin{tabular}{lll}
\hline Reversible Gates & Quantum cost & Types \\
\hline SG Gate(Sayem\& Ueda, 2010) & Unknown & $4 \times 4$ \\
BME Gate(Mahfuzzreza et al., 2013). & 5 & $4 \times 4$ \\
BSCL Gate (Rashmi et al., 2011) & Unknown & $6 \times 6$ \\
PAREEK Gate (Pareek et al., 2014) & 7 & $4 \times 4$ \\
RCQCA Gate (Misra et al., 2018). & 6 & $4 \times 4$ \\
URQG Gate (Bhoi et al., 2017). & Unknown & $3 \times 3$ \\
HCG Gate (James et al., 2007) & Unknown & $4 \times 4$ \\
HAS Gate (Misra et al., 2015). & 5 & $3 \times 3$ \\
FAS Gate (Misra et al., 2015). & 8 & $4 \times 4$ \\
OD Gate (Misra et al., 2015). & 10 & $5 \times 5$ \\
PPHCG Gate (James et al., 2007) & 6 & $4 \times 4$ \\
NG-PP Gate (Misra et al., 2017) & 5 & $5 \times 5$ \\
HG-PP Gate (Misra et al., 2017) & 4 & $5 \times 5$
\end{tabular}

\section{CONCLUSION}

In this paper, a survey of various works is carried out in the field of reversible logic with respect to reversible circuits which form the basic building block of quantum computers. This paper presents the reversible gates which are not shown in (Ankush \& Bhandari, 2016) and which are gathered from the literature till now.

\section{REFERENCES}

Abdesssied, N., Drechsher, R. (2016). Reversible and Quantum Circuits: Optimization and Complexity Analysis. Springer International publishing Switzerland. 1723.

Akbar, E. P. A., Haghparast, M., and Navi, K. (2011). Novel design of a fast reversible wallace sign multiplier circuit in nanotechnology. Microelectronics Journal, vol. 42, no. 8, 973-981

Ankush, \& Bhandari, A.S. (2016). Review Paper on Reversible Logic. International journal for Research in Electronics \& Electrical Engineering. 2(7), 1-6.

Bennett C.H. (1973) .Logical reversibility of Computation", IBM J. Research and Development, pp. 525-532

Bhoi, K.B., Misra, N.K., \& Pradhan, M. (2017). A Universal Reversible Gate Architecture for Designing $\mathrm{N}$ - Bit Comparator Structure in Quantum Dot Cellular Automata. International Journal of Grid and Distributed Computing. Vol. 10, no. 9. 33-46.

Biswas, P.K, Gupta, N., \& Patidar, N. (2014). Basic Reversible Logic Gates and It's Qca Implementation. (March 2017). International Journal of Engineering Research and Applications. 4(6), 12-16.

Garipelly, R., Kiran, M.P., \& Kumar, S.A. (2013). A Review on Reversible Logic Gates and their Implementation. International
The paper can further be extended towards the digital design development using reversible logic circuits which are helpful in quantum computing, low power CMOS, nanotechnology, cryptography, optical computing, DNA computing, digital signal processing (DSP), quantum dot cellular automata, communication, computer graphics, etc.

Journal of Emerging Technology and Advanced Engineering, vol. 3, no. 3, 417-423.

James, R.K., Shahana T. K., K. P. Jacob, K.P., \& S. Sasi, (2007). Fault Tolerant Error Coding and Detection usig Reversible Gates, IEEE TENCON, pp. 1- 4, 2007.

Landauer R (1961). Irreversibility and heat generation in the computingprocess. IBM J. Res. Dev., 5: 183-191.

Mahfuzzreza, M., Islam, R., and Ali, M. B.( 2013). Optimized Design of High Performance Reversible Multiplier using BME and MHNG Reversible Gate. American International Journal of Research in Science, Technology, Engineering and Mathematics, vol. 2, no. 2, 227-232.

Mamataj, S., Saha, D., \& Banu, N. (2014). A Review of Reversible Gates and its Application in Logic Design. American Journal of Engineering Research 2(4), 151-161.

Merkle, R.C., \& Drexler, K.E. (1996) "Helical logic", Nanotechnology, 7: pp. 325-339, 1996

Miller, D.M., Wille, R., Dueck, G.W.(2009). Synthesizing reversible circuits for irreversible functions. In Euromicro Conference on Digital System Design, Architectures, Methods and Tools. 749756. 
Special Conference Edition, November, 2019

Misra, N.K., Wairya, S., \& Singh, V.K. (2015). Frame of Reversible BCD Adder and Optimization Using New Reversible Logic Gates for Quantum Dot Cellular Automata. Australian Journal of Basic and Applied Sciences, 9(31). 286-298.

Misra, N.K., Wairya,S., \& Sen, B. (2017). Novel Conservative Reversible Error Control Circuits based Molecular QCA. International journal of Computer Applications in Technology. 2011.

Misra, N.K., Wairya, S., \& Sen, B. (2018). Design of Conservative Reversible Sequential Logic for Cost Efficient Emerging Nano circuits with enhanced Testability. Ain Shams Engineering journal, 2027-2037.

Mohammadi, M., and Eshghi, M. (2009). On figures of merit in reversible and quantum logic designs. Quantum Information Processing, vol. 8, no. 4, 297-318.

Murthy, M. K., Gayatri, G., and Kumar, R. M. (2012). Design of Efficient Adder Circuits Using Proposed Parity Preserving Gate (PPPG). International Journal of VLSI Design \& Communication Systems, vol. 3, no. 3, 83-93.

Pareek, V., Gupta, S., \& Jain, S. C. (2014). A Novel Realization of Sequential Reversible Building Blocks. In the 6th International Conference on Future Computational Technologies and Applications, 1-6.
Parhami, B. (2006). Fault Tolerant Reversible Circuits. Proc. ACSSC, pp. 1726-1729.

Rashmi S, Praveen, \& Tilak G. (1996) -Design of Optimized Reversible BCD Adder/Subtractor, IACSIT International Journal of Engineering and Technology, Vol.3, No.3.

Sayem, A. S. M., and Ueda, M. (2010). Optimization of Reversible Sequential Circuits. Journal of Computing, vol. 2, no. $6,208-214$.

Smoline, J., \& David P. (1996). "Five Two-Qubit Gates Are Sufficient To Implement The Quantum Fredkin Gate", Physics Review A, vol. 53, no.4, pp. 2855-2856,1996.

Soeken, M., Wille, R., Keszocze, O., Miller, D.M., \& Drechsler, R.(2015). Embedding of Large Boolean Functions for Reversible Logic. ACM Journal of Emerging Technology Computation System. 12(4).

Thapliyal, H. \& Ranganathan, N. (2010). Design of reversible sequential circuits optimizing quantum cost, delay and garbage outputs, ACM Journal of Emerging Technologies in Computing Systems, vol. 6, no.4, Article 14, pp. 14:1-14:35, Dec. 2010.

Yelekar, P.R., \& Chiwande, S.S. (2011). Introduction to Reversible Logic Gates \& Application. International journal of Computer Applications. 5-9. 